Pacific Journal of Mathematic 


\title{
COMPLEX INVERSION FOR THE GENERALIZED CONVOLUTION TRANSFORMATION
}

\author{
J. N. Pandey AND A. H. Zemanian
}

The complex inversion theory for the convolution transformation, which is due to Hirschman and Widder, is extended to certain generalized functions. This is accomplished by transferring the complex inversion formula onto the testing function space for the generalized function under consideration and then showing that the limiting process in the resulting formula converges with respect to the topology of the testing function space.

The Hirschman-Widder convolution transformation [1] has recently been extended to certain classes of generalized functions [2], and their real inversion formula [1; pp. 127-132] has been shown to be still valid when the limiting operation in that formula is understood as weak convergence in the space $\mathscr{D}^{\prime}$ of Schwartz distributions [3]. The purpose of the present work is to extend the complex inversion formula [1, Th. 7.1b, p. 231] in a similar way to the generalized convolution transformation.

The notation and terminology of this work follows that of [2]. $\mathscr{R}$ denotes the real one-dimensional euclidean space, and all testing functions herein are defined on $\mathscr{R}$. Throughout this work, $t$ and $x$ are variables in $\mathscr{R}$. A function that possesses continuous derivatives of all orders every-where on $\mathscr{R}$ is called smooth. If $f$ is a generalized function on $\mathscr{R}$, the notation $f(t)$, where $t \in \mathscr{R}$, is used merely to indicate that the testing functions, on which $f$ is defined, have $t$ as their independent variable; it does not mean that $f$ is a function of $t .\langle f, \varphi\rangle$ denotes the number assigned to some element $\varphi$ in a testing function space by a member $f$ of the dual space. Sometimes we write $\langle f(t), \varphi(t, x)\rangle_{t}$. This means that, for each fixed $x, \varphi(t, x)$ as a function of $t$ is a testing function to which the generalized function $f$ is being applied; the subscript $t$ is used to emphasize which variable is the independent variable for the testing functions of $f$. The $k$ th derivative of an ordinary or generalized function $f(t)$ is denoted alternatively by $D^{k} f, D_{t}^{k} f(t)$, or $f^{(k)}(t)$. Finally, $D$ is the space of smooth functions on $\mathscr{R}$ having compact supports. The topology of $D$ is that which makes its dual the space $D^{\prime}$ of Schwartz distributions on $\mathscr{R}[3 ; \mathrm{Vol}$. I, p. 65].

2. The spaces $\mathscr{L}_{c, d}$ and $\mathscr{L}_{c, d}^{\prime}$. The generalized functions appearing in this paper were discussed in $[2 ; \S 3]$. We briefly review their 
definition and paramount properties here.

Let $c$ and $d$ be two fixed real numbers, and let $\kappa_{c, d}(t)$ be a fixed smooth positive function defined on $-\infty<t<\infty$ such that

$$
\kappa_{c, d}(t)=\left\{\begin{array}{lc}
e^{c t} & 1<t<\infty \\
e^{d t} & -\infty<t<-1
\end{array}\right.
$$

$\mathscr{L}_{c, d}$ is defined as the linear space of all complex-valued smooth functions $\varphi(t)$ on $-\infty<t<\infty$ such that for each $k=0,1,2, \cdots$

$$
\gamma_{k}(\varphi)=\gamma_{c, d, l_{k}}(\varphi)=\sup _{-\infty<t<\infty}\left|\kappa_{c, d}(t) \varphi^{(k)}(t)\right|<\infty .
$$

We assign to $\mathscr{C}_{c, d}$ the topology generated by the collection of seminorms $\left\{\gamma_{k}\right\}_{k=0}^{\infty} . \quad \mathscr{C}_{c}, d$ is a sequentially complete Hausdorff locally convex topological linear space. Differentiation is a continuous linear mapping of $\mathscr{L}_{c, d}$ into itself.

The dual $\mathscr{L}_{c, d}^{\prime}$ of $\mathscr{L}_{c, d}$ is also sequentially complete. Under the customary definition of differentiation of generalized functions, namely,

$$
\left\langle f^{(1)}, \varphi\right\rangle=\left\langle f,-\varphi^{(1)}\right\rangle \quad f \in \mathscr{L}_{c, d}^{\prime}, \varphi \in \mathscr{L}_{c, d},
$$

differentiation is a continuous linear mapping of $\mathscr{L}_{c, d}^{\prime}$ into itself. The generalized functions with which we shall be concerned in this work, are the members of $\mathscr{C}_{c, d}^{\prime}$ for various choices of $c$ and $d$.

The restriction of any $f \in \mathscr{L}_{c, d}$ to $\mathscr{D}$ is in $\mathscr{D}^{\prime}$ because $\mathscr{D} \subset \mathscr{L}_{c, d}$ and the topology of $\mathscr{D}$ is stronger than that induced on it by $\mathscr{L}_{c, d}$. Similarly, if $c \leqq a$ and $b \leqq d$, then $\mathscr{L}_{a, b} \subset \mathscr{L}_{c, d}$, and the topology of $\mathscr{L}_{a, b}$ is stronger than that induced on it by $\mathscr{L}_{c, d}$. Consequently, the restriction of any $f \in \mathscr{L}_{c, d}^{\prime}$ to $\mathscr{L}_{a, b}$ is in $\mathscr{L}_{a, b}^{\prime}$.

3. The generalized convolution transformation. Let us first specify the type of kernel for which our complex inversion theory has been constructed. Let $s$ be a complex variable. Following Hirschman and Widder [1; p. 212], we set

$$
E(s)=\prod_{k=1}^{\infty}\left(1-\frac{s^{2}}{a_{k}^{2}}\right)
$$

where the $a_{k}$ are real numbers such that $0<a_{1} \leqq a_{2} \leqq a_{3} \leqq \cdots$ and

$$
\lim _{k \rightarrow \infty} \frac{k}{a_{k}}=\Omega \quad(0<\Omega<\infty) .
$$

Also, let

$$
G(z)=\frac{1}{2 \pi i} \int_{-i \infty}^{i \infty} \frac{e^{s z}}{E(s)} d s
$$$$
z=u+i v
$$ 
$G(z-t)$ will be the kernel of our convolution transformation.

Hirschman and Widder [1; pp. 213-214] have proven

Lemma 1. A. Let $\sigma=\operatorname{Re} s$ and $\tau=\operatorname{Im} s$. Then,

$$
|E(\sigma+i \tau)|=O\left(e^{\pi \Omega|\tau|}\right) \quad|\tau| \rightarrow \infty,
$$

and, for each $\eta>0$,

$$
|E(\sigma+i \tau)|^{-1}=o\left(e^{-\pi(\Omega-\eta)|\tau|}\right)
$$$$
|\tau| \rightarrow \infty,
$$

where both estimates hold uniformly for $\sigma$ in any finite interval.

B. $G(z)$ is an analytic function in the strip $|v|<\pi \Omega$, where $v=\operatorname{Im} z$.

C. Let $u=\operatorname{Re} z$, and let $\mu$ be the multiplicity of the zero of $E(s)$ occurring at the point $s=a_{1}$. Then,

$$
\begin{aligned}
& G(z)=p(z) e^{-a_{1} z}+R_{+}(z), \\
& G(z)=p(-z) e^{a_{1} z}+R_{-}(z),
\end{aligned}
$$

where $p(z)$ is a polynomial of degree $\mu-1$ and where, for each nonnegative integer $n$,

$$
\begin{array}{lr}
R_{+}^{(n)}(z)=O\left(e^{-\left(a_{1}+\varepsilon\right) u}\right) & u \rightarrow \infty, \\
R_{-}^{(n)}(z)=O\left(e^{\left(a_{1}+\varepsilon\right) u}\right) & u \rightarrow-\infty,
\end{array}
$$

for some $\varepsilon>0$, uniformly in every proper substrip $|v| \leqq \pi(\Omega-\eta)$ of the strip $|v|<\pi \Omega$.

By the above estimates on $G(z)$, if $c<a_{1}, d>-a_{1}$, and $z$ is a fixed point in the strip $|v|<\pi \Omega$, then $G(z-t)$ as a function of $t$ is in $\mathscr{L}_{c, d}$. Consequently, we can define the convolution transform $F(z)$ of a generalized function $f \in \mathscr{L}_{c, d}^{\prime}$ by

$$
F(z)=\langle f(t), G(z-t)\rangle \quad|\operatorname{Im} z|<\pi \Omega .
$$

Thus, under the stated restrictions on $c, d$, and $z$, our generalized convolution transformation maps $\mathscr{C}_{c, d}^{\prime}$ into the space of ordinary functions defined on the strip $|\operatorname{Im} z|<\pi \Omega$.

Theorem 1. Let $G(z)$ be defined by (2) and (3). Also, let $c<a_{1}$, $d>-a_{1}$, and $f \in \mathscr{L}_{c, d}^{\prime}$. If $F(z)$ is defined by $(4)$, then $F(z)$ is analytic on the strip $|\operatorname{Im} z|<\pi \Omega$, and

$$
F^{(1)}(z)=\left\langle f(t), G^{(1)}(z-t)\right\rangle \quad|\operatorname{Im} z|<\pi \Omega .
$$

Proof. Let $z$ be a fixed point of the strip $|\operatorname{Im} z|<\pi \Omega$. Also, let $C$ and $C_{1}$ be two concentric circles lying entirely within the said 
strip and having centers at $z$ and radii $r$ and $r_{1}$ respectively, where $0<r<r_{1}$. Finally, let $\Delta z$ be a complex increment such that $0<|\Delta z|<r$, and consider

$$
\frac{1}{\Delta z}[F(z+\Delta z)-F(z)]-\left\langle f(t), G^{(1)}(z-t)\right\rangle=\left\langle f(t), \theta_{\Delta_{z}}(t)\right\rangle
$$

where

$$
\theta_{\Delta z}(t)=\frac{1}{\Delta z}[G(z+\Delta z-t)-G(z-t)]-G^{(1)}(z-t) .
$$

Our theorem will be proven when we show that $\theta_{\Delta_{z}}(t) \rightarrow 0$ in $\mathscr{L}_{c, d}$ as $|\Delta z| \rightarrow 0$. By using Cauchy's integral formulas, we may write

$$
\begin{aligned}
\kappa_{c, d}(t) \theta_{\Delta z}^{(k)}(t)= & \frac{\kappa_{c, d}(t)(-1)^{k}}{2 \pi i} \\
& \times \int_{C_{1}} G^{(k)}(\zeta-t)\left[\frac{1}{\Delta z}\left(\frac{1}{\zeta-z-\Delta z}-\frac{1}{\zeta-z}\right)-\frac{1}{(\zeta-z)^{2}}\right] d \zeta \\
= & \frac{(-1)^{k} \Delta z}{2 \pi i} \int_{C_{1}} \frac{\kappa_{c, d}(t) G^{(k)}(\zeta-t)}{(\zeta-z-\Delta z)(\zeta-z)^{2}} d \zeta .
\end{aligned}
$$

By Lemma 1C, there exists a constant $B_{k}$ not depending on $t$ or $\zeta \in C_{1}$ such that

$$
\left|\kappa_{c, d}(t) G^{(k)}(\zeta-t)\right|<B_{k} \quad-\infty<t<\infty, \zeta \in C_{1} .
$$

Moreover, $|\zeta-z-\Delta z|>r_{1}-r$ and $|\zeta-z|=r_{1}$ if $\zeta \in C_{1}$. Therefore

$$
\gamma_{k}\left[\theta_{\Delta z}(t)\right] \leqq \frac{B_{k}|\Delta z|}{r_{1}\left(r_{1}-r\right)} \rightarrow 0 \quad|\Delta z| \rightarrow 0,
$$

which completes the proof.

4. Complex inversion. With $E(s)$ being defined by (2), set

$$
K(z)=\sum_{k=0}^{\infty} E^{(k)}(0) z^{-k-1} \text {. }
$$

It is a fact that $K(z)$ is analytic and single-valued in the $z$-plane slit along the imaginary axis from $-i \pi \Omega$ to $i \pi \Omega$. Moreover,

$$
E(s)=\frac{1}{2 \pi i} \int_{C} K(z) e^{s z} d z
$$

where $C$ is a closed rectifiable curve going in the positive direction around the segment $[-i \pi \Omega, i \pi \Omega]$ on the imaginary axis $[1 ; \mathrm{p} .223]$.

Now let $C_{\rho}$ be a closed rectifiable curve lying entirely within the strip $|\operatorname{Im} z|<\Omega \pi / \rho(0<\rho<1)$ and going in the positive direction around 
the segment $[-i \pi \Omega, i \pi \Omega]$ on the imaginary axis. The complex inversion formula for the convolution transform (4) is

$$
f(t)=\lim _{\rho \rightarrow 1-} \frac{1}{2 \pi i} \int_{C_{\rho}} K(z) F(t+\rho z) d z
$$

where weak convergence in $\mathscr{D}^{\prime}$ is understood. Note that, since $t$ is real, $0<\rho<1$, and $|\operatorname{Im} z|<\pi \Omega / \rho$, it follows from Theorem 1 that $F(t+\rho z)$ is analytic on $C_{\rho}$. Hence, the integral in (8) converges.

THEOREM 2. Let $E(s)$ be defined by (2), $G(z)$ by (3), and $K(z)$ by (6). Also, let $C_{\rho}$ be the curve described above. If $f \in \mathscr{L}_{c, d}^{\prime}$, where $c<a_{1}$ and $d>-a_{1}$, and if $F(z)$ is the convolution transform of $f$ in accordance with (4), then, for each $\varphi \in \mathscr{D}$ and as $\rho \rightarrow 1-$,

$$
\left\langle\frac{1}{2 \pi i} \int_{C_{\rho}} K(z) F(t+\rho z) d z, \varphi(t)\right\rangle \rightarrow\langle f, \varphi\rangle .
$$

This theorem will be proven by justifying the steps in the following manipulation:

$$
\begin{aligned}
& \left\langle\frac{1}{2 \pi i} \int_{C_{\rho}} K(z) F(t+\rho z) d z, \varphi(t)\right\rangle_{t} \\
= & \left\langle\varphi(t), \frac{1}{2 \pi i} \int_{C_{\rho}} K(z)\langle f(x), G(t+\rho z-x)\rangle_{x} d z\right\rangle_{t} \\
= & \frac{1}{2 \pi i} \int_{C_{\rho}} K(z)\left\langle\varphi(t),\langle f(x), G(t+\rho z-x)\rangle_{x}\right\rangle_{t} d z \\
= & \frac{1}{2 \pi i} \int_{C_{\rho}} K(z)\left\langle f(x),\langle\varphi(t), G(t+\rho z-x)\rangle_{t}\right\rangle_{x} d z \\
= & \left\langle f(x), \frac{1}{2 \pi i} \int_{C_{\rho}} K(z)\langle\varphi(t), G(t+\rho z-x)\rangle_{t} d z\right\rangle_{x} \\
& \quad \rightarrow\langle f(x), \varphi(x)\rangle
\end{aligned}
$$

First of all, by the analyticity of $K(z)$ and $F(t+\rho z)$, the integral on $C_{\rho}$ inside (10) is an analytic function of $t[4 ; p .99]$. Consequently, (10) has a meaning for $\varphi \in \mathscr{D}$ and is, in fact, an ordinary integration on $t$. That (10) is equal to (11) is obvious. Next, (11) becomes (12) upon interchanging the integrations on $t$ and $z$, a process that is justified by the facts that $\varphi(t)$ is smooth and of compact support. $K(z)$ is analytic on $C_{\rho}$, and $\langle f(x), G(w-x)\rangle$ is analytic for $|\operatorname{Im} w|<\pi \Omega$.

Our next objective is to show that (12) is equal to (13). Assume that the support of $\varphi(t)$ is contained in the closed finite interval $[A, B]$. For each $m=1,2,3, \cdots$, we partition $[A, B]$ into $m$ subintervals whose lengths are $\Delta t_{\nu, m}(\nu=1, \cdots, m)$. Let $\zeta_{\nu, m}$ be a point in the $\nu$ th subinterval, and assume that the maximum of the $\Delta t_{\nu, m}$ tends to 
zero as $m$ tends to infinity. Then,

$$
\begin{aligned}
& \left\langle\varphi(t),\langle f(x), G(t+\rho z-x)\rangle_{x}\right\rangle_{t}=\int_{A}^{B} \varphi(t)\langle f(x), G(t+\rho z-x)\rangle_{x} d t \\
& =\lim _{m \rightarrow \infty} \sum_{\nu=1}^{m} \varphi\left(\zeta_{\nu, m}\right)\left\langle f(x), G\left(\zeta_{\nu, m}+\rho z-x\right)\right\rangle \Delta t_{\nu, m} \\
& =\lim _{m \rightarrow \infty}\left\langle f(x), \sum_{\nu=1}^{m} \varphi\left(\zeta_{\nu, m}\right) G\left(\zeta_{\nu, m}+\rho z-x\right) \Delta t_{\nu, m}\right\rangle .
\end{aligned}
$$

If we can show that the sum within the last expression converges in $\mathscr{L}_{c, d}$ to $\int_{A}^{B} \varphi(t) G(t+\rho z-x) d t$ as $m \rightarrow \infty$, then the fact that $f \in \mathscr{L}_{c, d}^{\prime}$ will imply that (16) is equal to

$$
\langle f(x),\langle\varphi(t), G(t+\rho z-x)\rangle\rangle,
$$

which in turn will prove that (12) truly equals (13).

For any fixed $k=0,1,2, \cdots$, fixed $z \in C_{\rho}$, and fixed $\rho(0<\rho<1)$, set

$$
\begin{aligned}
A(x, m)= & \kappa_{c, d}(x) D_{x}^{k}\left[\sum_{\nu=1}^{m} \varphi\left(\zeta_{\nu, m}\right) G\left(\zeta_{\nu, m}+\rho z-x\right) \Delta t_{\nu, m}\right. \\
& \left.-\int_{A}^{B} \varphi(t) G(t+\rho z-x) d t\right] \\
= & (-1)^{k} \kappa_{\epsilon, d}(x)\left[\sum_{\nu=1}^{m} \varphi\left(\zeta_{\nu, m}\right) G^{(k)}\left(\zeta_{\nu, m}+\rho z-x\right) \Delta t_{\nu, m}\right. \\
& \left.-\int_{A}^{B} \varphi(t) G^{(k)}(t+\rho z-x) d t\right] ;
\end{aligned}
$$

differentiation under the integral sign is permissible here. We need merely show that $A(x, m)$ tends uniformly to zero on $-\infty<x<\infty$ as $m \rightarrow \infty$.

By virtue of the asymptotic properties of $G^{(k)}(t+\rho z-x)$ (Lemma 1C) and the conditions $c<a_{1}$ and $d>-a_{1}$, for an $\varepsilon>0$ we can choose $X$ so large that

$$
\left|\kappa_{c, d}(x) G^{(k)}(t+\rho z-x)\right|<\frac{\varepsilon}{3}\left[\int_{A}^{B}|\varphi(t)| d t\right]^{-1}
$$

for $A \leqq t \leqq B$ and $|x|>X$. Therefore, for $|x|>X$

$$
\left|\kappa_{c, d}(x) \int_{A}^{B} \varphi(t) G^{(k)}(t+\rho z-x) d t\right|<\frac{\varepsilon}{3}
$$

and

$$
\begin{array}{r}
\left|\kappa_{c, d}(x) \sum_{\nu=1}^{m} \varphi\left(\zeta_{\nu, m}\right) G^{(k)}\left(\zeta_{\nu, m}+\rho z-x\right) \Delta t_{\nu, m}\right| \\
<\frac{\varepsilon}{3}\left[\int_{A}^{B}|\varphi(t)| d t\right]^{-1} \sum_{\nu=1}^{m}\left|\varphi\left(\zeta_{\nu, m}\right)\right| \Delta t_{\nu, m} .
\end{array}
$$


We can now choose $m_{0}$ so large that for all $m>m_{0}$, the right-hand side of (18) is no greater than $2 \varepsilon / 3$. Thus, for $|x|>X$ and $m>m_{0}$, $|A(x, m)|<\varepsilon$; i.e., $A(x, m)$ tends uniformly to zero for $|x|>X$ as $m \rightarrow \infty$.

Now, $\varphi(t) G^{(k)}(t+\rho z-x)$ is a uniformly continuous function of $(t, x)$ on the domain $A \leqq t \leqq B$ and $-X \leqq x \leqq X$. Consequently, the quantity within the brackets on the right-hand side of (17) tends uniformly to zero on $-X \leqq x \leqq X$ as $m \rightarrow \infty$. The same is true for $A(x, m)$ because $\kappa_{c, d}(x)$ is bounded on $-X \leqq x \leqq X$. This completes the proof of the equality between (12) and (13).

That (13) is equal to (14) is proven in the same way. Indeed,

$$
\langle\varphi(t), G(t+\rho z-x)\rangle
$$

is also analytic for $|\operatorname{Im} \rho z|<\pi \Omega$ and possesses the same asymptotic properties as $x \rightarrow \pm \infty$ as does $G(t+\rho z-x)$. Consequently, we can partition the curve $C_{\rho}$ into $m$ arcs and proceed exactly as before.

The last step, namely that (14) tends to (15) as $\rho \rightarrow 1-$, is established by proving that

$$
\frac{1}{2 \pi i} \int_{C_{\rho}} K(z)\langle\varphi(t), G(t+\rho z-x)\rangle_{t} d z
$$

converges in $\mathscr{Z}_{c, d}^{2}$ to $\varphi(x)$ as $\rho \rightarrow 1-$. To do this, we first prove three lemmas.

This simple proof of the next lemma was suggested to the authors by the referee.

Lemma 2. Let $K(z)$ and $G(z)$ be defined as before, then

$$
\frac{1}{2 \pi i} \int_{C_{\rho}} K(z)\langle 1, G(t+\rho z-x)\rangle_{t} d z=1
$$

for all $-\infty<x<\infty$. (Here, 1 represents the function that is identically equal to 1 on $-\infty<t<\infty$.)

Proof. By Cauchy's theorem and Lemma 1C

$$
\langle 1, G(t+\rho z-x)\rangle_{t}=\int_{-\infty}^{\infty} G(t+\rho z-x) d t=1,
$$

while it follows from (7) with $s=0$ that

$$
\frac{1}{2 \pi i} \int_{C_{\rho}} K(z) d z=1,
$$

if $0<\rho<1$. 


$$
\mathrm{G}(\rho, t)=\frac{1}{2 \pi i} \int_{-i \infty}^{i \infty} \frac{E(\rho s)}{E(s)} e^{s t} d s .
$$

If $c<a_{1}, d>-a_{1}$, and $\delta>0$, then, as $\rho \rightarrow 1-, e^{-c t} G(\rho, t)$ tends uniformly to zero on $-\infty<t<-\delta$, and $e^{-d t} G(\rho, t)$ tends uniformly to zero on $\delta<t<\infty$.

Proof. We shall prove the assertion only for $e^{-c t} G(\rho, t)$ since the proof for $e^{-d t} G(\rho, t)$ differs in only trivial ways.

If $c \leqq 0$, then $e^{-c t} G(\rho, t)$ is nonnegative and monotonic increasing on $-\infty<t<0$ according to [1; Th. 4.2, p. 221]. Consequently, it converges uniformly to zero on $-\infty<t<-\delta$ as $\rho \rightarrow 1-$ since it converges to zero at $t=\delta[1$; Corollary 4.2, p. 222].

Next, assume that $0<c<a_{1}$. By [1; p. 220], for $|\operatorname{Re} s|<a_{1}$

$$
\int_{-\infty}^{\infty} e^{-s t} G(\rho, t) d t=\frac{E(\rho s)}{E(s)}
$$

We may differentiate (23) with respect to $s$ under the integral sign any number of times because each such differentiated integral converges uniformly in every compact subset of the open strip $|\operatorname{Re} s|<a_{1}$, in view of $[1 ;$ Th. $4.1 \mathrm{D}$, p. 219]. Hence,

$$
\int_{-\infty}^{\infty} t^{2} e^{-s t} G(\rho, t) d t=D_{s}^{2} \frac{E(\rho s)}{E(s)} .
$$

When $s$ is restricted to real values with $-a_{1}<s<a_{1}$, we may use logarithmic differentiation to compute $(24)$ [4; p. 16].

$$
\begin{aligned}
D_{s} \frac{E(\rho s)}{E(s)} & =\frac{E(\rho s)}{E(s)} D_{s} \log \frac{E(\rho s)}{E(s)} \\
& =\frac{E(\rho s)}{E(s)} D_{s} \sum_{k=1}^{\infty}\left[\log \left(a_{k}^{2}-\rho^{2} s^{2}\right)-\log \left(a_{k}^{2}-s^{2}\right)\right] \\
& =\frac{E(\rho s)}{E(s)} 2 s\left(1-\rho^{2}\right) \sum_{k=1}^{\infty} \frac{a_{k}^{2}}{\left(a_{k}^{2}-s^{2}\right)\left(a_{k}^{2}-\rho^{2} s^{2}\right)} .
\end{aligned}
$$

The term by term differentiation of a series used herein is valid because the result converges uniformly in compact subsets of

$$
-a_{1}<s<a_{1} .
$$

Another term by term differentiation, which is again valid, shows that

$$
D_{s}^{2} \frac{E(\rho s)}{E(s)}=\left(1-\rho^{2}\right) M(s, \rho)
$$

where the function $M(s, \rho)$ tends to a finite limit as $\rho \rightarrow 1-$ for every 
real $s$ such that $-a_{1}<s<a_{1}$.

Finally, choose the real number $\alpha$ such that $1<\alpha<a_{1} / c$. Then, since $G(\rho, t)$ is nonnegative and monotonic increasing on $-\infty<t<0$ [1; Th. 4.2, p. 221], for $t<0$

$$
\begin{aligned}
0 \leqq(\alpha-1) & \left(\frac{-t}{\alpha}\right)^{3} e^{-c t} G(\rho, t) \leqq \int_{t}^{t / \alpha} u^{2} e^{-c \alpha u} G(\rho, u) d u \leqq \int_{-\infty}^{\infty} u^{2} e^{-c \alpha u} G(\rho, u) d u \\
& =\left(1-\rho^{2}\right) M(c \alpha, \rho)
\end{aligned}
$$

or

$$
e^{-c t} G(\rho, t) \leqq \frac{\left(1-\rho^{2}\right) M(c \alpha, \rho)}{(\alpha-1)(-t / \alpha)^{3}} .
$$

By our previous results, this quantity tends uniformly to zero on $-\infty<t<\delta$ as $\rho \rightarrow 1-$, which is what we wished to show.

Lemma 4. Let $\left.\varphi \in \mathscr{D}, c<a_{1}, d\right\rangle-a_{1}$, and $G(\rho, t)$ be defined by (22). Then, for each fixed nonnegative integer $k$,

$$
N(x, \rho)=\kappa_{c, d}(x) \int_{-\infty}^{\infty}\left[\varphi^{(k)}(t)-\varphi^{(k)}(x)\right] G(\rho, t-x) d t
$$

tends to zero as $\rho \rightarrow 1$ - uniformly on $-\infty<x<\infty$.

Proof. We break up the integration in (28) into integrations on $-\infty<t<x-\delta, x-\delta<t<x+\delta$; and $x+\delta<t<\infty(\delta>0)$, and denote the corresponding quantities by $I_{1}(x, \rho), I_{2}(x, \rho)$, and $I_{3}(x, \rho)$ respectively.

$$
N(x, \rho)=I_{1}(x, \rho)+I_{2}(x, \rho)+I_{3}(x, \rho) .
$$

First of all, since $G(\rho, t-x)>0$ and $\int_{-\infty}^{\infty} G(\rho, t-x) d t=1[1 ; \mathrm{p}$. 219],

$$
\begin{aligned}
\left|I_{2}(x, \rho)\right| & =\left|\kappa_{c, d}(x) \int_{x-\delta}^{x+\delta}\left[\phi^{(k)}(t)-\varphi^{(k)}(x)\right] G(\rho, t-x) d t\right| \\
& \leqq \kappa_{c, d}(x) \sup _{x-\delta<t<x+\delta}\left|\phi^{(k)}(t)-\varphi^{(k)}(x)\right| \\
& \leqq \kappa_{c, d}(x) \delta \sup _{x-\delta<\tau<x+\delta}\left|\phi^{(k+1)}(\tau)\right| .
\end{aligned}
$$

Now, $\varphi$ is smooth and of compact support. Consequently, the last quantity is bounded by $\delta B$ where $B$ is a constant with respect to $x$ and $\delta$ when $\delta$ is restricted to $0<\delta<1$. Therefore, given an

$$
\varepsilon>0,\left|I_{2}(x, \rho)\right|
$$

is bounded by $\varepsilon$ for $\delta=\min (1, \varepsilon / B)$ and for $0<\rho<1$. Fix $\delta$ this way. 
Next, consider

$$
\begin{aligned}
I_{1}(x)= & \kappa_{c, d}(x) \int_{-\infty}^{x-\delta} \phi^{(k)}(t) G(\rho, t-x) d t \\
& -\kappa_{c, d}(x) \phi^{(k)}(x) \int_{-\infty}^{x-\delta} G(\rho, t-x) d t .
\end{aligned}
$$

Using the fact again that $G(\rho, t)$ is nonnegative everywhere, we may write

$$
\begin{aligned}
0 \leqq \int_{-\infty}^{x-\delta} G(\rho, t-x) d t & =\frac{1}{\delta^{2}} \int_{-\infty}^{-\delta} \delta^{2} G(\rho, y) d y \leqq \frac{1}{\delta^{2}} \int_{-\infty}^{-\delta} y^{2} G(\rho, y) d y \\
& \leqq \frac{1}{\delta^{2}} \int_{-\infty}^{\infty} y^{2} G(\rho, y) d y .
\end{aligned}
$$

According to [1; Th. $4.1 \mathrm{~A}$, p. 219$]$, the last quantity is equal to

$$
\delta^{-2} 2\left(1-\rho^{2}\right) \sum_{k=1}^{\infty} a_{k}^{-2}
$$

which tends to zero as $\rho \rightarrow 1-$. Since $\kappa_{c, d}(x) \varphi^{(k)}(x)$ is bounded for all $x$, this shows that the second term on the right-hand side of (29) converges uniformly to zero on $-\infty<x<\infty$ as $\rho \rightarrow 1-$.

Now, let $J_{1}(x)$ denote the first term on the right-hand side of (29), and assume that the support of $\varphi$ is contained in the closed finite interval $[A, B]$. For $-\infty<x-\delta \leqq A, J_{1}(x) \equiv 0$. On the other hand, for $A<x-\delta<\infty$, we have that $\kappa_{c, d}(x)<P e^{c x}$, where $P$ is a constant, and therefore

$$
\begin{aligned}
\left|J_{1}(x)\right| & \leqq P e^{c x} \int_{A}^{x-\delta}\left|\varphi^{(k)}(t)\right| e^{c(t-x)} e^{-c(t-x)} G(\rho, t-x) d t . \\
& \leqq P \int_{A}^{B}\left|\varphi^{(k)}(t)\right| e^{c t} d t \sup _{-\infty<t-x<-\delta}\left|e^{-c(t-x)} G(\rho, t-x)\right| .
\end{aligned}
$$

By Lemma 3, the right-hand side tends to zero uniformly on $-\infty<x<\infty$ as $\rho \rightarrow 1-$.

Thus, we have shown that $I_{1}(x)$ tends to zero uniformly on $-\infty<x<\infty$. A similar argument demonstrates that $I_{3}(x)$ does the same as $\rho \rightarrow 1-$. Altogether then, we have proven that

$$
\varlimsup_{\rho \rightarrow 1-}|N(x, \rho)| \leqq \varepsilon \quad-\infty<x<\infty,
$$

and, since $\varepsilon$ is arbitrary, our lemma is proven.

We are finally ready to prove that (14) tends to (15) as $\rho \rightarrow 1-$. For any fixed nonnegative integer $k$, consider,

$$
\kappa_{c, d}(x) D_{x}^{k}\left[\frac{1}{2 \pi i} \int_{c_{\rho}} K(z)\langle\varphi(t), G(t+\rho z-x)\rangle_{t} d z-\varphi(x)\right] .
$$

Because $\varphi \in \mathscr{D},\langle\varphi(t), G(t+\rho z-x)\rangle_{t}$ is a continuous functions of $(z, x)$ 
for $z \in C_{\rho}$ and $-\infty<x<\infty$, and an analytic function of $x$ for every $z \in C_{\rho}$. Consequently, we may interchange the differentiations with respect to $x$ with the integration on $C_{\rho}[4 ; \mathrm{p} .99]$, and then interchange it once again with the integration on $t$. Some integrations by parts then show that (30) is equal to

$$
\kappa_{c, d}(x) \frac{1}{2 \pi i} \int_{C_{\rho}} K(z)\left\langle\varphi^{(k)}(t), G(t+\rho z-x)\right\rangle_{t} d z-\kappa_{c, d}(x) \phi^{(k)}(x) .
$$

By using [1; Th. 6.1a, p. 226], this expression can be rewritten as

$$
\begin{gathered}
\kappa_{c, d}(x) \int_{-\infty}^{\infty}\left[\varphi^{(k)}(t)-\varphi^{(k)}(x)\right] G(\rho, t-x) d t \\
+\kappa_{c, d}(x) \phi^{(k)}(x)\left[\frac{1}{2 \pi i} \int_{c_{\rho}} K(z)\left\langle 1, G(t \rho z-x)_{t} d z-1\right] .\right.
\end{gathered}
$$

By virtue of Lemmas 2 and 4 , (31) tends uniformly to zero on $-\infty<x<\infty$ as $\rho \rightarrow 1-$. Hence, the testing function in (14) truly converges in $\mathscr{C}_{c, d}$ to $\varphi(x)$, and our proof of Theorem 2 is complete. This theorem can be proved even without using Lemma 2.

\section{REFERENCES}

1. I. I. Hirschman, Jr. and D. V. Widder, The Convolution Transform, Princeton University Press, Princeton, New Jersey, 1955.

2. A. H. Zemanian, A generalized convolution iransformation, J. SIAM Appl. Math. 15 (1967), 324-346.

3. L. Schwartz, Théorie des distributions, vols. I and II, Hermann, Paris, 1957 and 1959.

4. E. C. Titchmarsh, Theory of Functions, second edition, Oxford University Press, 1939.

Received December 20, 1966. This work was supported by the Air Force Cambridge Research Laboratories, Bedford, Massachusetts, under Contract AF 19(628)-2981.

University of Alberta

AND

State University of New York at Stony Brook 



\section{PACIFIC JOURNAL OF MATHEMATICS}

\section{EDITORS}

H. ROYDEN

Stanford University

Stanford, California

\section{J. P. Jans}

University of Washington

Seattle, Washington 98105

\section{J. DUGUNDJI}

Department of Mathematics University of Southern California Los Angeles, California 90007

\section{RICHARD ARENS}

University of California

Los Angeles, California 90024

\section{ASSOCIATE EDITORS}

E. F. BECKENBACH

B. H. NEUManN

F. WOLF

K. YosIDA

\section{SUPPORTING INSTITUTIONS}

UNIVERSITY OF BRITISH COLUMBIA CALIFORNIA INSTITUTE OF TECHNOLOGY UNIVERSITY OF CALIFORNIA MONTANA STATE UNIVERSITY UNIVERSITY OF NEVADA NEW MEXICO STATE UNIVERSITY OREGON STATE UNIVERSITY UNIVERSITY OF OREGON OSAKA UNIVERSITY UNIVERSITY OF SOUTHERN CALIFORNIA
STANFORD UNIVERSITY

UNIVERSITY OF TOKYO UNIVERSITY OF UTAH WASHINGTON STATE UNIVERSITY UNIVERSITY OF WASHINGTON AMERICAN MATHEMATICAL SOCIETY CHEVRON RESEARCH CORPORATION TRW SYSTEMS NAVAL WEAPONS CENTER 


\section{Pacific Journal of Mathematics}

\section{Vol. 25, No. $1 \quad$ September, 1968}

Glen Eugene Bredon, Cosheaves and homology................... 1

Robin Ward Chaney, A chain rule for the transformation of integrals in

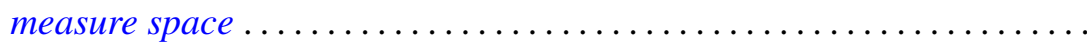

Colin W. Clark, On relatively bounded perturbations of ordinary differential operators................................... 59

John Edwin Diem, A radical for lattice-ordered rings.............. 71

Zeev Ditzian, On a class of convolution transforms ................ 83

Dennis Garoutte and Paul Adrian Nickel, A note on extremal properties characterizing weakly $\lambda$-valent principal functions............. 109

Shwu-Yeng Tzeng Lin, Fixed point properties and inverse limit spaces . . . 117

John S. Lowndes, Some dual series equations involving Laguerre polynomials ................................. 123

Kirti K. Oberai, Sum and product of commuting spectral operators ....... 129

J. N. Pandey and Armen H. Zemanian, Complex inversion for the generalized convolution transformation..................... 147

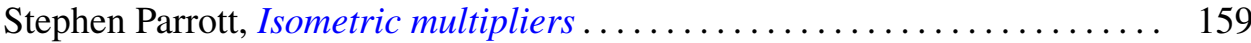

Manoranjan Prasad, Note on an extreme form .................. 167

Maciej Skwarczyński, A representation of a bounded function as infinite product in a domain with Bergman-Shilov boundary surface ......... 177

John C. Taylor, The Šilov boundary for a lattice-ordered semigroup ...... 185 Donald Reginald Traylor and James Newton Younglove, On normality and pointwise paracompactness ............................. 193

L. Tzafriri, Quasi-similarity for spectral operators on Banach spaces ... 